Planetary Systems in the Universe - Observation, Formation and Evolution

Proceedings IAU Symposium No. 202, (C)2004 IAU

Alan Penny, Pawel Artymowicz, Anne-Marie Lagrange, 6 Sara Russell, eds.

\title{
The dust composition of discs surrounding isolated Herbig Ae/Be stars
}

\author{
G. Meeus \\ Astronomical Institute, K.U. Leuven, Celestijnenlaan 200B, B-3001 \\ Leuven, Belgium
}

J. Bouwman, L.B.F.M. Waters

Astronomical Institute "Anton Pannekoek", University of Amsterdam, Kruislaan 403, NL-1098 SJ Amsterdam, The Netherlands

C. Waelkens

Astronomical Institute, K.U. Leuven, Celestijnenlaan 200B, B-3001

Leuven, Belgium

M.E. van den Ancker

Harvard-Smithsonian Center for Astrophysics, 60 Garden Street, $M S$ 42, Cambridge, MA 02138, USA

\begin{abstract}
We present Infrared Space Observatory (ISO) spectra of 14 isolated Herbig $\mathrm{Ae} / \mathrm{Be}$ stars. The IR spectra were combined with photometric data from the UV to the sub-mm region. We defined two key groups, based upon the spectral shape of the IR region. The results can be summarized as follows (see also Meeus et al. 2001): (1) the continuum of the IR to sub-mm region can be reconstructed by the sum of a power-law and a cool component, which can be represented by a black body. Possible locations for these components are an optically thick, geometrically thin disc (power-law component) and an optically thin flared region (black body); (2) remarkably, some sources lack the silicate bands; (3) PAH bands are present in at least $50 \%$ of our sample; with one exception, PAHs are not present in sources which only show a power-law continuum in the IR; (4) the dust in HAEBE stars shows strong evidence for coagulation; this dust processing is unrelated to any of the central star properties.
\end{abstract}

\section{Introduction}

A circumstellar (CS) disc is expected to be a natural byproduct of the starforming process (e.g. Shu et al 1987). This theoretical expectation has obtained wide support from observations. The CS disc is expected and observed to gradually disappear, but remnants are still found around several Main-Sequence (MS) stars, such as Vega. The discs around young stars are assumed to be the site of planet formation. Herbig Ae/Be stars (HAEBEs) are the more massive analogs of $\mathrm{T}$ Tauri stars (Herbig 1960). They are seen as the progenitors of Vega-type stars, and have the following characteristics: (1) large IR excesses due to thermal re-emission of CS dust, (2) spectral emission lines due to CS gas, and (3) masses between 2 and $8 \mathrm{M}_{\odot}$. 


\section{Targets and Observations}

The objects we selected are fourteen so-called isolated HAEBE stars. These HAEBE stars are not located inside a star-forming region, but show all the other characteristics of a HAEBE star and are presumably the somewhat more evolved members of the HAEBE group.

The sources have been observed with the ISO Short Wavelength Spectrometer (SWS), covering an interval from 2 to $45 \mu \mathrm{m}$. Photometric (UV to sub-mm) data were collected from the literature and a Spectral Energy Distribution (SED) was composed for each star; it was found that: (1) the near-IR excess is similar for all stars in our sample, (2) sub-mm excess is substantial around all stars, and (3) the mid-IR flux shows large source to source differences.

\subsection{Overall appearance and classification of the SEDs}

Despite large diversity in the 10 to $100 \mu \mathrm{m}$ spectral appearance, it is possible to describe the SEDs of all stars with 3 spectral components (see Fig. 1): (1) a power-law, (2) solid state bands and (3) a black body. For some sources, it is even possible to fit the dust continuum with only a power-law. We have accordingly classified the sample:

1. Group I contains sources for which the continuum can be reconstructed by a power-law and a black body

- Group Ia sources show solid state bands

- Group Ib sources are without solid state bands

2. Group II sources only need a power-law to fit their continuum

- Group IIa sources show solid state bands

- Group IIb sources are without solid state bands

\section{Geometry of the disc and its effects on the SED}

From a comparison between group I and II sources, we conclude that: (1) the amount of mid-IR excess is dominant and rising for group I sources but moderate and rather descending for group II sources; (2) the total IR luminosity is also different: $\mathrm{L}_{\mathrm{IR}} / \mathrm{L}_{*}$ is on average 0.38 and 0.19 for group I and II respectively, while the maximum value for a non-flared disc is 0.25 (Kenyon \& Hartmann 1987); (3) solid state bands are present with similar average strength in group Ia and IIa sources.

The classification of our sample of HAEBE stars can be explained with the following simple physical picture (see Fig. 1): (1) a geometrically thin disc being responsible for the power-law continuum. Hot dust in the innermost part of the disc is responsible for the near-IR excess; (2) a flared region of warm dust around the thin disc, causing a rising continuum in the IR; (3) an optically thin inner disc/disc atmosphere causes the solid state bands.

This disc geometry has also been proposed for TTS (Chiang \& Goldreich, 1997). One can explain the SEDs of group II sources by assuming that the inner part (component I lower-right panel Fig. 1) is partially optically thick, shielding 

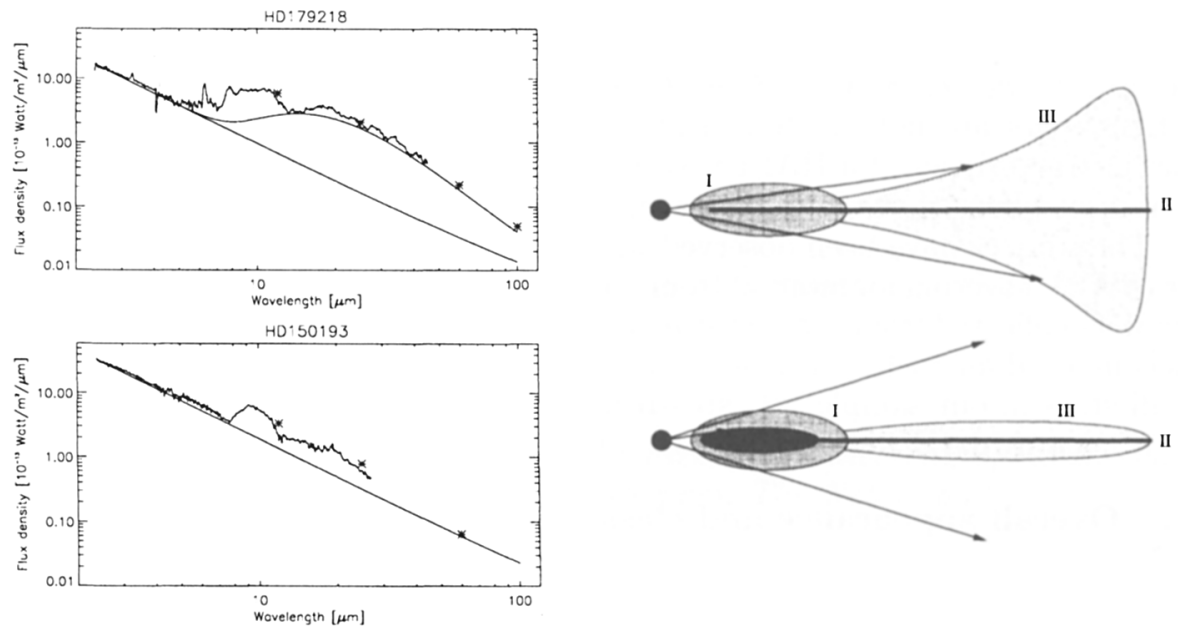

Figure 1. Left: IR spectra of a group I source (up) and a group II source (down); the different components are indicated. Right: schematics of the model; a flared disc (up) and a non-flared disc (down).

the outer parts of the disc from direct stellar radiation, what prevents the disc from flaring.

\section{Conclusions}

1. The near-IR spectral region is very similar $\longleftrightarrow$ similar inner disc structure and composition of the dust, while there is a highly diverse spectral appearance between 10 to $100 \mu \mathrm{m}$

2. The SEDs can be decomposed into 3 components:

(1) a power-law (optically thick disc)

(2) solid state bands (small hot grains)

(3) black-body (flared disc component)

3. PAH emission correlates with the presence of a flaring disc component

4. Silicate bands are absent in some sources: evidence for large grains?

\section{References}

Chiang, E.I., \& Goldreich, P. 1997, ApJ, 490, 368

de Graauw, M.S., Haser, L.N., Beintema, M.A. et al. 1996, A\&A, 315, L49

Herbig, G.H. 1960, ApJS, 4, 337

Kenyon, S.J., \& Hartmann L. 1987, ApJ, 323, 714

Meeus, G., Waters, L.B.F.M., Bouwman et al. 2001, A\&A, 365, 476

Shu, F.H., Adams, F.C., \& Lizano, S., 1987, ARA\&A, 25, 23 